\title{
Antimitochondrial Antibodies and Reactivity to $N$. Aromaticivorans Proteins in Icelandic Patients with Primary Biliary Cirrhosis and Their Relatives
}

Sigurdur Olafsson, M.D., Hallgrimur Gudjonsson, M.D., Carlo Selmi, M.D., Ph.D., Katsushi Amano, M.D., Ph.D., Pietro Invernizzi, M.D., Ph.D., Mauro Podda, M.D., and M. Eric Gershwin, M.D. Department of Internal Medicine, Landspitali University Hospital, Reykjavik, Iceland; Division of Rheumatology, Allergy and Clinical Immunology, University of California at Davis, School of Medicine, Davis, California; Division of Internal Medicine, Department of Medicine, Surgery and Dentistry, San Paolo School of Medicine, University of Milan, Italy

OBJECTIVES: $\quad$ Primary biliary cirrhosis (PBC) is a chronic, progressive cholestatic disease of unknown etiology characterized by serum antimitochondrial antibodies (AMA) directed against a functionally related family of mitochondrial enzymes. We recently suggested that $N$. aromaticivorans might be the trigger of autoimmunity in PBC. No data are available on the specificity and crossreactivity of AMA in a genetically homogenous group of patients, such as the Icelandic population.

METHODS: $\quad$ To address these issues and to confirm previous findings in a unique population, we obtained sera from 14 PBC patients and 85 first-degree relatives, all of Icelandic descent. We analyzed such sera for AMA specificity using recombinant mitochondrial antigens and for reactivity against $N$. aromaticivorans proteins.

RESULTS: $\quad$ Thirteen of the 14 Icelandic patients with PBC (93\%) were found AMA positive. We found that 5/13 AMA positive sera (38\%) reacted against PDC-E2 only; 5/13 (or 38\%) reacted against BCOADC-E2; and $2 / 13(15 \%)$ reacted against all three antigens. There was no reactivity against OGDC-E2. Reactivities of patients' sera against $N$. aromaticivorans were consistent with the AMA status. One serum among the 85 first-degree relatives (1.2\%) was found to be AMA-positive, as well as reactive against $N$. aromaticivorans.

CONCLUSIONS: Interestingly, despite the homogenous genetic background, the group of Icelandic patients with PBC was heterogeneous in their AMA reactive patterns and also reacted with $N$. aromaticivorans proteins.

(Am J Gastroenterol 2004;99:1-4)

\section{INTRODUCTION}

Iceland is an island located in the North Atlantic with a population of over 280,000 , according to 2002 estimates, with limited degrees of migration. Because of its location and history, $94 \%$ of the current population is of Icelandic descent, i.e., homogeneous mixture of descendants of Scandinavians and Celts, with only $6 \%$ being of foreign origin. Such demographic background makes this population unique as it is considered to present a high degree of genetic homogeneity $(1,2)$.

Primary biliary cirrhosis (PBC) is an autoimmune disease of unknown etiology leading to progressive destruction of small intrahepatic bile ducts (3) and characterized by female predominance and serum autoantibodies to mitochondrial antigens (AMA) (3). The reported prevalence rates of PBC among relatives of affected individuals suggest an individual predisposition to the disease (4). Genetic background appears important in determining susceptibility $(5,6)$, along with a number of additional factors, including molecular mimicry by microbes $(7,8)$ and/or xenobiotics $(9,10)$. One hypothesis is, therefore, that environmental factors trigger $\mathrm{PBC}$ in genetically predisposed individuals (11). AMA are directed against epitopes within the components of the 2-oxo acid dehydrogenase complex (2-OADC). From a clinical standpoint, we note that, as the vast majority of AMA-positive individuals (12) already present or will develop signs of PBC, AMA seropositivity is considered a highly specific as well as sensitive marker for the disease, especially when techniques are based on recombinant mitochondrial antigens (Ags), rather than indirect immunofluorescence (IIF) (13). Finally, we have recently demonstrated that sera from patients with $\mathrm{PBC}$ recognize proteins from a ubiquitous xenobiotics-metabolizing bacterium, $N$. aromaticivorans (8). We also showed that such 
Table 1. Clinical Characteristics of Icelandic Patients with PBC and Their Relatives

\begin{tabular}{lcc}
\hline & $\begin{array}{c}\text { PBC } \\
(\mathrm{n}=14)\end{array}$ & $\begin{array}{c}\text { Relatives }^{\ddagger} \\
(\mathrm{n}=85)\end{array}$ \\
\hline Female sex & $14(100 \%)$ & $39(46 \%)$ \\
Icelandic ethnicity & $14(100 \%)$ & $85(100 \%)$ \\
Age at enrollment (yr)* & $59 \pm 11$ & $48 \pm 17$ \\
Age at diagnosis (yr)* & $50 \pm 11$ & - \\
Advanced histological stage (III-IV) & $7(50 \%)$ & - \\
\hline
\end{tabular}

${ }^{*}$ Continuous variables are expressed as mean \pm standard deviation,

${ }^{\ddagger}$ First-degree relatives of patients with PBC include parents $(\mathrm{n}=10)$, siblings $(\mathrm{n}=45)$, and children $(\mathrm{n}=30)$.

reactivity was 100 to 1000 -fold stronger when compared to E. $\operatorname{coli}(8)$.

The aim of this study was to investigate, using highly sensitive and specific methods, AMA specificities of sera from patients with $\mathrm{PBC}$ and first-degree relatives belonging to the Icelandic population. Moreover, in order to confirm our previous findings in a geographically well-distinct population, we investigated the reactivity of the same sera against proteins from $N$. aromaticivorans.

\section{MATERIALS AND METHODS}

\section{Subjects}

We obtained serum samples from 14 patients with wellestablished diagnosis of PBC according to internationally accepted criteria (14), as well as from 85 first-degree relatives (parents, siblings, and offspring) without any known sign of liver disease. Table 1 shows the characteristics of patients and relatives enrolled in this study. All subjects were of Icelandic descent. All patients with $\mathrm{PBC}$ were receiving ursodeoxycholic acid at the moment of serum sampling. There were 85 first-degree relatives, including 10 parents, 45 siblings, and 30 children. All sera were tested for AMA by ELISA and for reactivity against $N$. aromaticivorans proteins by Western blotting.

\section{Determination of $A M A$}

The AMA status of PBC patients was investigated by ELISA using recombinant molecules of the triple hybrid antigen $\left(\mathrm{pMIT}_{3}\right)$, pyruvate dehydrogenase complex (PDC-E2), 2-oxo glutarate dehydrogenase complex (OGDC-E2), and branched-chain 2-oxo acid dehydrogenase complex
(BCOADC-E2), as previously described (13, 15). Sera reacting against one or more of these Ags were considered to be AMA-positive using our previous definition.

\section{Immunoblotting against Bacterial Antigens}

Bacterial cultures were obtained as previously described (8). Two hundred micrograms of proteins from bacterial lysate were loaded onto a $10 \%$ SDS-PAGE gel $(10 \times 10 \mathrm{~cm})$ and electrophoresed at $20 \mathrm{~mA}$ for $2 \mathrm{~h}$. Proteins were transferred onto nitrocellulose membranes and blocked with 5\% powdered milk. Membrane strips were incubated with patients' and relatives' sera diluted $10^{-3}$. After washing, strips were incubated with a horseradish peroxidase (HRP)-conjugated goat antihuman Ig (Zymed Laboratories, Inc., South San Francisco, CA) for $1 \mathrm{~h}$. The washed blots were developed using Super Signal Substrate (Pierce, Rockford, IL) and exposed to X-ray film.

Human PDC-E2 specific mouse-derived IgG monoclonal antibodies (clones 4C8) $(13,16)$, and rabbit antilipoic acid antisera (17) were also studied by Western blots using HRPconjugated goat antimouse or antirabbit IgG (Zymed Laboratories, Inc.) at a $10^{-4}$ dilution as secondary antibody. Known positive and negative sera were included as controls throughout the study.

\section{RESULTS}

Patients with PBC were classified by AMA status using recombinant mitochondrial auto-Ags and ELISA, since these antigens have been shown to be the most sensitive and specific substrate for the detection of AMA (13). AMA patterns with different antigens in our study population are shown in Table 2. Of the 14 Icelandic patients with $\mathrm{PBC}$, there were 13 patients who were AMA positive and 1 that was AMA negative. Five out of 13 sera presented antibodies against PDC-E2 only, 5/13 against PDC-E2 and BCOADC-E2; 2/13 against PDC-E2, OGDC-E2; and BCOADC-E2, and 1/13 reacted only with the triple hybrid. Among relatives, $1 / 85$ serum $(1.2 \%)$ was found to be AMA-positive with reactivity to all three mitochondrial antigens (i.e., PDC-E2, OGDC-E2, and BCOADC-E2); such serum had been obtained from the father of an AMA-positive subject with PBC.

Figure 1 shows the patterns of positivity observed with the $14 \mathrm{PBC}$ sera (P1-P14) against protein homogenates from

Table 2. AMA Patterns among Patients with $\mathrm{PBC}$ and Relatives

\begin{tabular}{|c|c|c|c|c|}
\hline & \multicolumn{3}{|c|}{$\mathrm{PBC}$} & \multirow{2}{*}{$\begin{array}{l}\text { Relatives } \\
(\mathrm{n}=85)\end{array}$} \\
\hline & Total $(\mathrm{n}=14)$ & Stages I-II $(\mathrm{n}=7)$ & Stages III-IV $(\mathrm{n}=7)$ & \\
\hline AMA positive & 13 (93\%) & $7(100 \%)$ & $6(86 \%)$ & 0 \\
\hline Anti-MIT ${ }_{3}$ & $13(93 \%)$ & $7(100 \%)$ & $6(86 \%)$ & 0 \\
\hline Anti-PDC-E2 only & $5(36 \%)$ & $2(29 \%)$ & $3(43 \%)$ & 0 \\
\hline Anti PDC-E2 and BCOADC-E2 & $5(36 \%)$ & $2(29 \%)$ & $3(43 \%)$ & 0 \\
\hline Anti-PDC-E2, BCOADC-E2, and OGDC-E2 & $2(14 \%)$ & $2(29 \%)$ & 0 & $1 / 85(1 \%)$ \\
\hline AMA negative & $1(7 \%)$ & 0 & $1(14 \%)$ & 0 \\
\hline
\end{tabular}




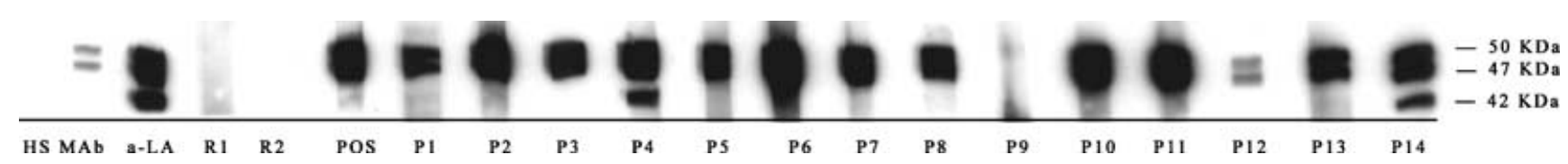

Figure 1. Lysates of $N$. aromaticivorans were separated by PAGE, transferred to nitrocellulose, and probed with different primary antibodies. The figure shows the lack of reactivity observed with one healthy subject serum (HS) and two AMA-negative relatives' sera (R1, R2). Further, the reactivities against the bacterial 50,47, and $42 \mathrm{kDa}$ proteins are shown for a specific antihuman PDC-E2 murine monoclonal antibody (mAb), a rabbit antilipoic acid polyclonal serum (a-LA), one known AMA-positive PBC serum (POS), and 14 Icelandic PBC sera (P1-P14). Note that P9, the only AMA-negative PBC serum, also failed to react against any bacterial protein.

N. aromaticivorans. All AMA-positive patients with PBC $(13 / 13)$ reacted with two proteins (molecular weights 47 and $50 \mathrm{kDa}$ ) from $N$. aromaticivorans. Among them, three patients also reacted against a $42 \mathrm{kDa}$ protein. No reactivity was observed among PBC sera against the previously described $60 \mathrm{kDa}$ and $49 \mathrm{kDa}$ proteins. Eighty-five first-degree relatives of patients with $\mathrm{PBC}$ were also studied and the one AMA-positive subject was also found to have antibodies to $N$. aromaticivorans. This patient is currently clinically healthy and asymptomatic. Eighty-four out of eighty-five sera from relatives did not react against the bacterial proteins.

\section{DISCUSSION}

The pathogenesis of $\mathrm{PBC}$ remains poorly understood; in particular, genetic factors explaining the susceptibility or the different progression rates have been widely investigated in the past decades $(5,6)$. The study of variants of the major histocompatibility complex (MHC), for example, despite being strongly associated with other autoimmune conditions, has not demonstrated a universal correlation with PBC. Among others, the typing of the genetic variants of $\operatorname{HLA}-\mathrm{II}(18,19)$, as well as of TNF- $\alpha$ (20) in patients with PBC, have lead to conflicting results.

Seropositivity to AMA is a powerful tool in the diagnostic challenge often represented by patients with intrahepatic cholestasis, although its sensitivity through IIF fails to identify $100 \%$ of cases of PBC (3). The mapping of the autoepitope recognized by AMA within a complex of mitochondrial enzymes has represented a critical observation in the study of the pathogenesis of the disease (21). Previous observations of AMA reactivity in other populations indicated that patients' sera reacted with one or more components of the 2-OADC pathway, including PDC-E2, BCOADC-E2, and/or OGDC-E2. In fact, patients retain their individual antigenic reactive profile throughout the course of disease. It is thus unclear whether the different AMA reactive pattern reflects genetic differences or merely stochastic events secondary to disease initiation. Interestingly, this latter possibility has been described in the past for the raise of autoantibodies in experimental models of autoimmune disease, where a simple genetic model failed to explain the prevalence of anti-Sm antibodies in the offspring of inbred anti-Sm-positive mice (22).The ideal experiment to address these differences would be to study AMA reactivity in a genetically identical population (i.e., large number of monozygotic twins). The closest homologous situation is an environment such as Iceland that not only presents a founder effect but also a significant degree of inbreeding. We freely admit the limitations of this assumption but also note the uniqueness of this population and the ability to test not only the AMA reactive patterns but also this population reactivity to $N$. aromaticivorans. Recently, our group has demonstrated the highly specific and sensitive crossreactivity between PBC sera and five proteins from this ubiquitous bacterium capable of metabolizing certain xenobiotics (8). Our previous results, obtained in patients with PBC from Italy and the United States, indicated that $N$. aromaticivorans is the best-known etiologic candidate for molecular mimicry in PBC.

Iceland has a population of approximately 280,000 and unique historical features that make this country particularly valuable for evaluating the role of genetics leading to different phenotypes in complex diseases such as PBC. Although the genetic homogeneity of Icelanders has been recently debated based on factors such as the heterozygosity of autosomal markers (23) or mitochondrial DNA variations (24), it is generally accepted that some degree of a founder effect, started with the first settlements from Norway about 1,130 yr ago, and the presence of a number of highly inbred families are characteristics of this region.

With regards to $\mathrm{PBC}$, limited epidemiological data on the prevalence or incidence of the disease in Iceland are available. The only report ever published was a 1994 retrospective study that demonstrated a prevalence rate for PBC in 1992 of nine cases per 100,000 (25), compatible with rates from other Scandinavian countries ranging between 28 and 152 cases per million (26). If such numbers were to be considered currently applicable to Iceland, we might expect the number of individuals affected by PBC to range between 5 and 28 in 2002. In this study we obtained serum samples from 14 patients with $\mathrm{PBC}$, and we believe that, although not including all Icelandic patients, our series is considered highly representative of patients in the Reykjavik area.

We investigated AMA reactivities of sera from patients with $\mathrm{PBC}$ and first-degree relatives using the most sensitive method available (13). Our data failed to identify a unique AMA pattern among patients. The highly represented reactivity against PDC-E2 is in accordance with other observations on larger series of patients (27). We identified one 
AMA-positive asymptomatic relative among the 85 included in our study. This is somewhat lower than previous data that found that up to $6 \%$ of family members are AMA-positive or present "familial PBC" (26). However, the series described herein may be not large enough to comply with such prediction or estimates. Our data on the reactivity of patients' and relatives' sera against $N$. aromaticivorans confirm our previous findings in the U.S. and Italian populations. We note that no reactivity was observed against two previously described bacterial proteins (of molecular weights 60 and $49 \mathrm{kDa}$ ) (8), although this lack of reactivity may be due to the small number of patients studied and to the limited prevalence of reactivity to such Ags. However, the two bacterial Ags (47 and $50 \mathrm{kDa}$ ) previously shown to be recognized by $100 \%$ of anti-PDC-E2-positive sera (8), were also reactive against all the AMA-positive sera included in the present study. In conclusion, based on the results obtained from an Icelandic population of patients with PBC and their relatives, we suggest that AMA specificities are the results of environmental stimuli, rather than only genetic predisposition, as observed for susceptibility to the disease and similar to studies of PBC in monozygotic twins (28).

Reprint requests and correspondence: M. Eric Gershwin, M.D., Division of Rheumatology, Allergy and Clinical Immunology, University of California at Davis, TB 192, Davis, CA 95616.

Received May 14, 2004; accepted May 21, 2004.

\section{REFERENCES}

1. Gulcher JR, Jonsson P, Kong A, et al. Mapping of a familial essential tremor gene, FET1, to chromosome 3q13. Nat Genet 1997; 17:84-7.

2. Jonsson H, Steinsson K. Rheumatology in Iceland. Br J Rheumatol 1997;36:577-9.

3. Neuberger J. Primary biliary cirrhosis. Lancet 1997;350: 875-9.

4. Tsuji K, Watanabe Y, Van De Water J, et al. Familial primary biliary cirrhosis in Hiroshima. J Autoimmun 1999;13:1718.

5. Tanaka A, Borchers AT, Ishibashi H, et al. Genetic and familial considerations of primary biliary cirrhosis. Am J Gastroenterol 2001;96:8-15.

6. Agarwal K, Jones DE, Bassendine MF. Genetic susceptibility to primary biliary cirrhosis. Eur J Gastroenterol Hepatol 1999;11:603-6.

7. Van de Water J, Ishibashi H, Coppel RL, et al. Molecular mimicry and primary biliary cirrhosis: Premises not promises. Hepatology 2001;33:771-5.

8. Selmi C, Balkwill DL, Invernizzi P, et al. Patients with primary biliary cirrhosis react against a ubiquitous xenobioticmetabolizing bacterium. Hepatology 2003;38:12507.
9. Long SA, Van de Water J, Gershwin ME. Antimitochondrial antibodies in primary biliary cirrhosis: The role of xenobiotics. Autoimmun Rev 2002;1:37-42.

10. Leung PS, Quan C, Park O, et al. Immunization with a xenobiotic 6-bromohexanoate bovine serum albumin conjugate induces antimitochondrial antibodies. J Immunol 2003; 170:5326-32.

11. Jones DE. Pathogenesis of primary biliary cirrhosis. J Hepatol 2003;39:639-48.

12. Neuberger J, Bradwell AR. Anti-mitochondrial antibodies in primary biliary cirrhosis. J Hepatol 2002;37:712-6.

13. Miyakawa H, Tanaka A, Kikuchi K, et al. Detection of antimitochondrial autoantibodies in immunofluorescent AMAnegative patients with primary biliary cirrhosis using recombinant autoantigens. Hepatology 2001;34:243-8.

14. Kaplan MM. Primary biliary cirrhosis. N Engl J Med 1996;335:1570-80.

15. Moteki S, Leung PS, Coppel RL, et al. Use of a designer triple expression hybrid clone for three different lipoyl domain for the detection of antimitochondrial autoantibodies. Hepatology 1996;24:97-103.

16. Migliaccio C, Nishio A, Water J, et al. Monoclonal antibodies to mitochondrial E2 components define autoepitopes in primary biliary cirrhosis. J Immunol 1998;161:5157-63.

17. Sasaki M, Ansari A, Pumford N, et al. Comparative immunoreactivity of anti-trifluoroacetyl (TFA) antibody and anti-lipoic acid antibody in primary biliary cirrhosis: Searching for a mimic. J Autoimmun 2000;15:51-60.

18. Donaldson P, Agarwal K, Craggs A, et al. HLA and interleukin 1 gene polymorphisms in primary biliary cirrhosis: Associations with disease progression and disease susceptibility. Gut 2001;48:397-402.

19. Invernizzi P, Battezzati PM, Crosignani A, et al. Peculiar HLA polymorphisms in Italian patients with primary biliary cirrhosis. J Hepatol 2003;38:401-6.

20. Donaldson PT. TNF gene polymorphisms in primary biliary cirrhosis: A critical appraisal. J Hepatol 1999;31:366-8.

21. Mackay IR, Whittingham S, Fida S, et al. The peculiar autoimmunity of primary biliary cirrhosis. Immunol Rev 2000;174:226-37.

22. Eisenberg RA, Craven SY, Warren RW, et al. Stochastic control of anti-Sm autoantibodies in MRL/Mp-lpr/lpr mice. J Clin Invest 1987;80:691-7.

23. Arnason E, Sigurgislason H, Benedikz E. Genetic homogeneity of Icelanders: Fact or fiction? Nat Genet 2000;25:373-4.

24. Arnason E. Genetic heterogeneity of Icelanders. Ann Hum Genet 2003;67:5-16.

25. Gunnarson R, Gudjonsson H, Jonasson JG, et al. Primary biliary cirrhosis in Iceland. Iceland Med J 1994;80:93.

26. Parikh-Patel A, Gold E, Mackay IR, et al. The geoepidemiology of primary biliary cirrhosis: Contrasts and comparisons with the spectrum of autoimmune diseases. Clin Immunol 1999;91:206-18.

27. Leung PS, Coppel RL, Ansari A, et al. Antimitochondrial antibodies in primary biliary cirrhosis. Semin Liver Dis 1997;17:61-9.

28. Selmi C, Mayo MJ, Bach N, et al. Primary biliary cirrhosis in monozygotic and dizygotic twins: Genetics, epigenetics, and environment. Gastroenterology 2004;127:485-92. 